\title{
Evaluación de una intervención proambiental en escolares de educación primaria (10-13 años) de Castilla-La Mancha (España)
}

\author{
Evaluation of a Proenvironmental Intervention in Primary School students (10-13 age) from \\ Castilla-La Mancha (Spain)
}

Avaliação de uma ação pró ambiental em estudantes do ensino fundamental (10-13 anos) em Castilla-La Mancha (Espanha)

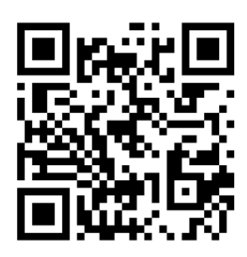

Belén Torres

Universidad de Castilla-La Mancha

Toledo, España

belentorrestorrijos@gmail.com

https://orcid.org/0000-0003-0039-3492

Universidad de Castilla-La Mancha

Toledo, España

maria.amerigo@uclm.es

(iD) https://orcid.org/0000-0002-8831-9221

Juan Antonio García

Universidad de Castilla-La Mancha

Talavera de la Reina, España

juan.garcia@uclm.es

https://orcid.org/0000-0002-3693-6180

Recibido • Received • Recebido: 02 / 10 / 2019

Corregido • Revised • Revisado: 06 / 06 / 2021

Aceptado • Accepted • Aprovado: 25 / 08 / 2021

\begin{abstract}
Resumen:
Introducción. La actual crisis ambiental revela la necesidad de actuar inmediatamente desde la educación ambiental a edades tempranas. El presente trabajo presenta un estudio de caso cuyo objetivo es comprobar si una intervención educativa es adecuada para fomentar actitudes proambientales, conductas y conocimientos relativos a la correcta separación de residuos que influyan positivamente sobre el cuidado del entorno. Metodología. Mediante un experimento de campo con un diseño intergrupal de dos grupos aleatorios (experimental y control) se aplicó un cuestionario antes y después de una intervención proambiental en el aula a 75 estudiantes de educación primaria. Se miden la actitud proambiental, la percepción de conducta doméstica de reciclaje y el nivel de conocimiento sobre separación de residuos. Se analizan la fiabilidad de las medidas y la efectividad de la intervención mediante ANOVAs con medidas repetidas. Resultados. Los resultados indican que tras la intervención el grupo experimental $(\mathrm{N}=38)$-en comparación con el
\end{abstract}


http://doi.org/10.15359/ree.25-3.11

http://www.una.ac.cr/educare

educare@una.ac.cr

grupo control ( $\mathrm{N}=37)$ - aumentó su actitud proambiental, su percepción de la conducta doméstica de reciclaje y su nivel de conocimiento sobre separación de residuos; lo que demostraría la eficacia de la intervención proambiental realizada. Discusión. La intervención didáctica propuesta fue diseñada para generar un mayor conocimiento de lo que se podría denominar como conducta ecológica responsable, centrada en el reciclaje, especialmente efectiva a la hora de incrementar la actitud proambiental relacionada con la dimensión de eco-conocimiento.

Palabras claves: Educación ambiental; actitud; conducta; conocimiento; educación básica.

\begin{abstract}
:
Introduction. The current environmental crisis reveals the need to act immediately from the field of environmental education from a very early age. This paper presents a case study that aims to verify the efficiency of an educational intervention to promote pro-environmental attitudes, perception of household recycling behaviors, and knowledge related to proper waste disposal. Method. By means of a field experiment with an intergroup design of two randomized groups (experimental and control), a questionnaire was conducted before and after a pro-environmental intervention in a classroom with 75 primary education students. Pro-environmental attitude, perception of household recycling behavior, and level of knowledge about waste disposal at home were evaluated. The reliability of the measures and the effectiveness of the intervention were analyzed using repeatedmeasures ANOVA. Results. The results reveal that, after the intervention, the experimental group ( $\mathrm{N}$ $=38$ ) -in comparison with the control group ( $\mathrm{N}=37)$ - increased its pro-environmental attitude, its perception of the household recycling behavior, and its level of knowledge about waste disposal; this would demonstrate the effectiveness of the conducted pro-environmental intervention. Discussion. The proposed educational intervention was designed to generate more knowledge of what could be called responsible ecological behavior, focused on recycling, being especially effective in increasing pro-environmental attitudes related to the eco-knowledge dimension.
\end{abstract}

Keywords: Environmental education; attitude; behavior; knowledge; basic education.

\title{
Resumo:
}

Introdução. A atual crise ambiental revela a necessidade de atuar de imediato na educação ambiental desde os primeiros anos de vida. O presente trabalho apresenta um estudo de caso cujo objectivo é verificar se uma ação de sensibilização a nível escolar é essencial para fomentar atitudes pró ambientais, comportamentos e conhecimentos relativos à correta separação dos resíduos que esclareçam de os cuidados necessario com o meio ambiente. Metodologia. Por meio de uma experiência de campo com uma concepção intergrupal de dois grupos aleatórios (experimental e de controlo) foi aplicado um questionário antes e depois de uma ação de esclarecimento pró ambiental numa sala de aula a 75 alunos do ensino fundamental. Identificou-se a atitude pró ambiental, a perceção de comportamentos domésticos de reciclagem e o nível de conhecimento sobre a separação dos resíduos. A fiabilidade das medidas e a eficácia da intervenção foram analisadas utilizando ANOVAs para medidas repetidas. Resultados. Os resultados indicam que após a ação de esclarecimento, o grupo experimental ( $\mathrm{N}=38)$, em comparação com o outro grupo ( $\mathrm{N}=37$ ), aumentou a sua atitude pró ambiental, sua perceção de comportamentos domésticos de reciclagem e o seu nível de conhecimento sobre a separação de resíduos; o que demonstra a eficácia da intervenção pró ambiental realizada. Discussão. A intervenção didática proposta foi projetada para gerar mais 
conhecimento ao que poderia ser chamado de comportamento ecológico responsável, focado na reciclagem, sendo especialmente eficaz no aumento da atitudes pró ambiental relacionada à dimensão do eco-conhecimento.

Palavras-chave: Educação ambiental; atitude; comportamento; conhecimento; educação básica.

\section{Introducción}

De acuerdo con diversas personas autoras, las actividades humanas ejercen un impacto significativo en la actual crisis ambiental (Paula-Acosta et al., 2019; Severiche-Sierra et al., 2016). Los avances de la sociedad hacia la industrialización han ocasionado una pérdida de la conexión humana con la naturaleza, la cual se deriva en la disminución de la conciencia ambiental y del respeto hacia el medio ambiente (White et al., 2017). Sin embargo, la mayor presencia de la problemática ambiental en la vida cotidiana de la ciudadanía ha provocado un creciente interés respecto a la exigencia de aceptar el papel del ser humano en la situación crítica del planeta e intervenir con el fin de revertir las consecuencias ambientales causadas por la actividad antropogénica (Vanegas-Rico et al., 2018). Autoras como Lima y Branco (2018) sostienen la necesidad de modificar los comportamientos individuales que conllevan el deterioro de los recursos naturales, con el fin de afrontar los problemas ambientales que acucian nuestro planeta.

Observando la situación específica de España, un estudio del Centro de Investigaciones Sociológicas (CIS, 2010) afirma que el $65,2 \%$ de los sujetos encuestados está bastante o muy preocupado por los temas relativos al medio ambiente. Sin embargo, debemos tener en cuenta que esta mayor preocupación por el medio ambiente no siempre se traduce en la práctica de conductas proambientales (López-Miguens et al., 2014). Esta baja relación entre las actitudes proambientales y los comportamientos consecuentes viene determinada por diversos factores; pero antes de señalarlos sería conveniente delimitar conceptualmente las principales variables implicadas en el presente estudio. El concepto de actitudes proambientales o preocupación ambiental, en sentido amplio y según la consideración establecida por Dunlap y Jones (2002), implicaría un extenso rango de fenómenos que irían desde la conciencia sobre las consecuencias negativas de los problemas ambientales hasta el apoyo a la protección medioambiental. Según estos estudios, la preocupación ambiental se expresa y se operativiza a través de constructos teóricos típicos de la investigación sobre actitudes y sus componentes cognitivos, afectivos y conativos, lo que Dunlap y Jones (2002) denominan aproximación teorética. Es decir, la preocupación ambiental se expresaría a través de las conocimientos, creencias y valores; los sentimientos, y las intenciones de conducta sobre las cuestiones medioambientales. Por lo que respecta a la conducta proambiental, cabría definirla como aquella que beneficia al ambiente o lo daña lo menos posible (Steg y Vlek, 2009), caracterizada porque se realiza con el propósito de proteger los recursos naturales; deliberada y voluntaria; responde a requerimientos sociales e individuales y se fundamenta en la conciencia y el conocimiento medioambiental (Hernández y Suárez, 2006). 
http://doi.org/10.15359/ree.25-3.11

http://www.una.ac.cr/educare

educare@una.ac.cr

La investigación psicosocial respecto a las relaciones entre actitudes y conductas permite analizar el ámbito concreto del medio ambiente y entender el porqué de la baja correlación observada entre actitudes proambientales y comportamientos ecológicos o, más específicamente, el bajo porcentaje de varianza en este tipo de comportamientos explicado por las actitudes proambientales, tal y como se mencionaba al comienzo del párrafo anterior. Respecto a esto último, uno de los modelos más utilizados es la teoría $A B C$ (" $A$ " de actitud; " $B$ " de behavior, conducta, en inglés; $\mathrm{y}$ " $\mathrm{C}$ " de contexto, que incluiría factores tecnológicos, económicos, físicos, institucionales y sociales) propuesta por Stern (2000). Según esta teoría, cuanto más difíciles de ejecutar, más tiempo consuman o más caras sean las conductas proambientales, menor será su dependencia de factores actitudinales y mayor de los factores contextuales. En este sentido, una persona puede mostrar una alta preocupación ambiental por la contaminación del aire provocada por el tráfico y mostrar una actitud muy favorable a la compra de un vehículo eléctrico, pero el alto coste económico que supone (factor contextual) hace inviable su comportamiento. Es importante promover actitudes proambientales entre la población, de ahí la importancia de la educación ambiental porque, sin duda, serán determinantes en la ejecución de comportamientos ecológicos; pero es necesario analizar también los factores contextuales concretos ya que estos también resultan determinantes en la conducta y pueden minimizar el impacto de las actitudes y hacer inoperante una intervención en el ámbito educativo, si no se tienen en cuenta.

Si nos detenemos en el caso concreto del municipio en el que se encuentra el centro educativo donde se lleva a cabo este estudio, podemos observar una difícil situación ambiental y ecológica. A pesar de que el ayuntamiento de la localidad gestiona adecuadamente los residuos urbanos y domésticos, el reciclaje de estos mismos, y potencia el cuidado de las zonas verdes y el arbolado urbano, la interacción de quienes habitan el municipio con el entorno no es adecuada, y se causa una problemática ambiental difícil de resolver sin la participación ciudadana. De ello surge el interés en llevar a cabo el presente trabajo, por lo que fue necesario evaluar el contexto ambiental de la localidad seleccionada y, posteriormente, de acuerdo con lo recomendado por Flórez-Yepes et al. (2018), adaptar los instrumentos y la intervención a las necesidades ecológicas y el estado del municipio.

El combate contra el cambio climático y la defensa del medio ambiente constituyen algunos de los objetivos de desarrollo sostenible reiteradamente defendidos por la Organización de las Naciones Unidas a través del Programa de las Naciones Unidas para el Desarrollo (PNUD, 2015). De acuerdo con Moreno-Fernández (2017), es necesario abordar aspectos relativos a la contaminación y el respeto al medio ambiente en el ámbito educativo, en concreto en la etapa de educación primaria; con el fin de sensibilizar y concienciar al alumnado sobre el problema ambiental existente en el planeta, fomentar sus actitudes proambientales y así contribuir 
http://doi.org/10.15359/ree.25-3.11

positivamente en su implicación en el cuidado del entorno (Jiménez-Denis et al., 2017; White et al., 2017). Por ello, la educación ambiental se concibe como una herramienta imprescindible a la hora de incentivar el compromiso de las futuras generaciones en la preservación del medio ambiente (Ordóñez-Diaz et al., 2018), al impulsar, a través del aprendizaje, la reflexión del estudiantado sobre la necesidad inminente de realizar cambios en las actitudes y conductas individuales de los seres humanos; así como ampliar sus conocimientos para promover la toma de una posición activa en la solución y prevención de problemáticas ambientales (SevericheSierra et al., 2016).

Ante un panorama en el que numerosos estudios reconocen la importancia del papel de la educación ambiental en la concienciación social sobre la problemática del planeta y el cambio climático (Galvis Riaño et al., 2019), poseen especial relevancia las intervenciones didácticas que se realizan en el marco de la educación para el cuidado del medio ambiente (Cardoso et al., 2018). Algunas investigaciones han puesto de manifiesto el impacto positivo de dichas intervenciones sobre el aprendizaje y las percepciones del alumnado respecto a diversos aspectos ambientales y consecuencias del cambio climático (Thorn y Bogner, 2018; White et al., 2017). Sin embargo, algunas otras insisten en la importancia de ofrecer, a la población escolar, recursos didácticos e intervenciones educativas que la involucren en sus actitudes y acciones cotidianas, pues la única forma de influir en la realidad ambiental presente es modificar, en cada individuo, la forma de actuar sobre el entorno (Demoly y Santos, 2018).

Autores como Cerrillo (2010) aseguran que los relatos y las narraciones son recursos didácticos que cuentan con numerosas ventajas asociadas a la formación del estudiantado, considerados instrumentos de transmisión de valores (Etxaniz, 2011). En este sentido, existen experiencias previas que señalan la idoneidad de trabajar con historias diseñadas ad hoc para promover la adquisición de habilidades en escolares (More, 2012), indican su influencia positiva en el fomento de conductas y actitudes proambientales; así como en la ampliación de los conocimientos de escolares sobre asuntos relacionados con el medio ambiente y en el impulso de cambios positivos en su conciencia y su relación con el entorno (Mahasneh et al., 2017).

Passafaro y Livi (2017) sostienen la necesidad de crear intervenciones educativas centradas en comportamientos ecológicos específicos relacionados con habilidades y conocimientos complejos, tales como la separación de residuos; enfatiza la importancia de conectar las intervenciones que se realicen en el aula con la vida cotidiana de la población estudiantil y sus necesidades reales, con el fin de crear un impacto significativo y duradero en las actitudes, conductas y conocimientos ambientales del estudiantado (Torres Rivera et al., 2016).

El presente trabajo plantea como principal objetivo comprobar si una intervención didáctica realizada en el aula produce cambios favorables en las actitudes proambientales del alumnado, así como en su percepción de la conducta doméstica de reciclaje y su nivel 
http://doi.org/10.15359/ree.25-3.11

http://www.una.ac.cr/educare

educare@una.ac.cr

de conocimiento respecto a la correcta separación de residuos. Para lograr estos objetivos se diseñó un estudio de caso con una metodología cuasiexperimental, mediante un experimento de campo, donde se formularon las siguientes hipótesis:

H1-La actitud proambiental de la población escolar es más elevada tras la intervención didáctica.

H2- La percepción de la población escolar sobre la conducta de separación de residuos en el ámbito doméstico es superior después de la intervención didáctica.

H3- El nivel de conocimiento relativo a la separación de residuos mejora tras la intervención didáctica.

\section{Metodología \\ Participantes}

La muestra se compone de 75 escolares pertenecientes a los cursos de $5^{\circ}$ y $6^{\circ}$ de educación primaria de un centro público situado en una localidad castellanomanchega (Torrijos, España), de quienes 40 eran niñas (53,3\%) y 35 eran niños (46,7\%). El rango de edad fue entre los 10 y 13 años, la edad media de 11,03 años (DT =0,73).

La muestra fue de tipo casual o incidental y seleccionada por conveniencia. Una vez conocido el número de escolares que participarían en el estudio, se dividió la totalidad de la muestra en un grupo experimental que fue sometido a la intervención didáctica (38 estudiantes) y un grupo control que no fue sometido a la intervención didáctica (37 participantes). Esta división se realizó de forma aleatoria; no se observaron diferencias significativas en las variables objeto de estudio entre el grupo experimental y el de control en el momento preintervención.

Al tratarse de escolares menores de edad, padres, madres o personas tutoras autorizaron su participación voluntaria y anónima en el estudio, al igual que la dirección del centro. El estudio cumplió la confidencialidad y los valores éticos requeridos en la investigación con seres humanos.

\section{Instrumento}

Se diseñó un cuestionario autoadministrado integrado por cuatro secciones que fue previamente validado por ocho personas expertas en el ámbito educativo.

En primer lugar, se incluyó la escala de medición de la actitud proambiental (EAPROAMB) en edad escolar, propuesta por Moreno et al. (2016). Esta escala mide distintos aspectos del cuidado y la protección medioambiental categorizados en dos dimensiones: (1) "eco-afinidad" o respeto por la naturaleza, medida a través de 8 ítems; y (2) "eco-conocimiento" o eco- 
http://doi.org/10.15359/ree.25-3.11

responsabilidad que hace referencia al comportamiento ecológico responsable, medida a través de 6 ítems. Todos los ítems de esta escala están formulados siguiendo el tipo Likert con cinco opciones de respuesta (desde $1=$ "Muy en desacuerdo" hasta $5=$ "Muy de acuerdo") e incluye emoticonos asociados a cada opción para facilitar la respuesta. La elección de esta escala se debe a que Moreno et al. (2016) diseñan y aplican la EAPROAMB a escolares de entre 10 y 13 años de España (mismo tramo etario y país que el considerado en el presente estudio de caso). Además, en su trabajo aplican un proceso metodológico riguroso para el diseño de la escala, partiendo de una sólida revisión teórica de los constructos implicados que se complementa con un estudio piloto; reportan una adecuada fiabilidad para las dos dimensiones consideradas; y proporcionan evidencias empíricas de una estructura bidimensional recurriendo a un análisis factorial confirmatorio.

En segundo lugar, con el fin de conocer la percepción de la conducta de separación de residuos en el ámbito doméstico, se diseñó una medida formada por cuatro ítems que incluía una pregunta genérica de separación de residuos en el hogar y tres cuestiones específicas referidas al reciclaje, según los diferentes tipos de contenedores que ofrece el consistorio municipal a la ciudadanía para la separación de residuos, tomando como referencia y preguntando en este caso sobre (1) vidrio; (2) cartón y papel; y (3) plásticos y envases. Estos ítems se respondían según tres categorías de respuesta en función de la apreciación de la frecuencia de separación de residuos en el hogar (Nunca, A veces o Siempre). Este formato de respuesta estuvo inspirado por el estudio llevado a cabo por el Centro de Investigaciones Sociológicas (CIS, 2010) para medir en la población española la frecuencia de realización de diferentes conductas proambientales. Para los análisis estadísticos posteriores, se calculó un índice de conducta percibida de separación de residuos para cada individuo que recogía la diferencia en términos porcentuales entre el número de ítems a los que el sujeto había respondido "siempre" y "nunca". La puntuación en este índice oscilaba entre 100 (si había respondido "siempre" a los cuatro ítems) y -100 (si había respondido "nunca" a estos mismos).

En tercer lugar, se incorporó una pregunta con el fin de evaluar el nivel de conocimiento que los escolares y las escolares poseían respecto a una adecuada separación de residuos en distintos contenedores (" ¿Crees que podrías enseñar a otras personas cómo separar los residuos en distintos contenedores? Inténtalo uniendo cada residuo con el contenedor adecuado donde desecharlo"). Así, se presentaban a las personas participantes un total de doce residuos diferentes (orgánicos, papel o cartón, vidrio y plásticos o envases), los cuales debían vincular con el contenedor en el que depositarían dichos residuos. La variable que medía el conocimiento de quienes participaron sobre la separación de residuos se calculó computando el número de respuestas correctas y expresando el resultado en una escala de 0 a 10.

Para finalizar, se incluyeron algunas variables sociodemográficas, tales como el sexo, el curso y la edad. 
http://doi.org/10.15359/ree.25-3.11

http://www.una.ac.cr/educare

educare@una.ac.cr

\section{Intervención didáctica}

La intervención realizada con los niños y las niñas que componían el grupo experimental estaba formada por tres actividades claramente diferenciadas: aproximación al problema ambiental; exposición de un caso específico adecuado a la edad del grupo de escolares; y ejecución de un taller de separación de residuos. El diseño fue validado por docentes de similares niveles educativos a quienes participaron en el estudio.

De esta forma, en primer lugar, se realizó un debate reflexivo con el gran grupo, en el cual se trataron actitudes del ser humano que poseen una influencia negativa sobre el medio ambiente (deforestación, problema de escasez de agua asociado a su desperdicio, caza de animales salvajes o en peligro de extinción, medios de transporte privados y contaminación, construcción de carreteras en espacios protegidos, etc.), así como actitudes de los seres humanos que protegen y conservan el entorno natural (empleo de energías renovables, respeto por los seres vivos y su hábitat natural, separación de residuos, medios de transporte públicos y movilidad sostenible, etc.).

A continuación, se procedió a la lectura en gran grupo de una narración diseñada ad hoc teniendo en cuenta las características evolutivas de las personas participantes. El relato contaba la historia de cómo, a través de la cooperación, unos niños y niñas eran capaces de solucionar un gran problema ambiental que asediaba su ciudad. La narración se elaboró exprofeso por dos motivos fundamentales: la necesidad de adecuar la historia a las edades concretas de los escolares y las escolares participantes y el interés de que esta reflejase un problema observable en el municipio (suciedad e inadecuada gestión de la basura) para fomentar la implicación del alumnado en la intervención. Tras la lectura del relato, se lanzaron algunas preguntas a quienes participaron sobre el contenido de la historia y su paralelismo con el municipio de los escolares y las escolares.

Por último, se realizó un taller de separación de residuos. Para ello, se dividió a las personas participantes en pequeños grupos, asignando a cada persona una bolsa de basura con residuos en su interior. Miembros de cada grupo debían decidir conjuntamente en qué contenedor depositarían cada residuo y, en el momento de desecharlo, explicar de qué residuo se trataba y por qué habían elegido ese contenedor para tirarlo.

La intervención empleada en el presente estudio se realizó en el aula de referencia de aquellas personas participantes que formaban parte del grupo experimental, y se contó con la duración de una sesión escolar de 60 minutos aproximadamente.

\section{Procedimiento}

Para la consecución de los objetivos, se planteó un experimento de campo con un diseño intergrupal de dos grupos aleatorios (experimental y control) y recogida de información en dos momentos (pre-post intervención) con un intervalo de tres semanas. El cuestionario descrito se administró en el aula en la que tenían lugar las sesiones lectivas.

8 
http://doi.org/10.15359/ree.25-3.11

http://www.una.ac.cr/educare educare@una.ac.cr

El análisis de los datos se realizó con los paquetes IBM ${ }^{\circledR}$ SPSS $^{\circledR}$ Statistics 24.0 y R 3.1.1.

\section{Resultados}

\section{Fiabilidad de las medidas}

Teniendo en cuenta la naturaleza ordinal o dicotómica de los ítems utilizados para la medición de los diferentes conceptos, y siguiendo las recomendaciones de Viladrich et al. (2017), se calculó el omega ordinal $(\omega O)$ de las tres variables implicadas: actitudes ambientales (diferenciando entre eco-afinidad y eco-conocimiento); conducta percibida de separación de residuos en el hogar; y grado de conocimiento sobre la separación de residuos. En la Tabla 1 se presentan los resultados obtenidos para este análisis de la fiabilidad junto con los estadísticos descriptivos en ambos momentos. Como se puede observar, el $\omega O$ en la fase pre-test osciló entre .89 y .93 , mientras que en el post-test se situó entre .73 y .96, lo que indica que las escalas tenían una adecuada consistencia interna.

Tabla 1: Estadísticos descriptivos y fiabilidad de las medidas de actitud, conducta y conocimiento $(N=75)$

\begin{tabular}{lccccccccccc}
\hline Constructo & \multicolumn{4}{c}{ Pre-test } & \multicolumn{1}{c}{ Post-test } \\
\cline { 2 - 12 } & $M$ & $D T$ & Min & Max & $\omega O$ & $M$ & $D T$ & Min & Max & $\omega O$ \\
\hline Eco-afinidad & 2.86 & 1.06 & 1.38 & 5.00 & .92 & 3.74 & 1.18 & 1.38 & 5.00 & .96 \\
Eco-conocimiento & 3.38 & 0.88 & 2.00 & 5.00 & .89 & 4.07 & 0.99 & 1.83 & 5.00 & .94 \\
$\begin{array}{l}\text { Conducta percibida de } \\
\text { separación de residuos }\end{array}$ & -41.00 & 57.49 & -100.00 & 100.00 & .93 & -3.33 & 62.10 & -100.00 & 100.00 & .92 \\
$\begin{array}{l}\text { Conocimiento sobre } \\
\text { separación de residuos }\end{array}$ & 6.92 & 1.15 & 3.33 & 8.33 & .89 & 8.02 & 1.30 & 4.17 & 10.00 & .73 \\
\hline
\end{tabular}

Nota: Elaboración propia.

\section{Análisis de la efectividad de la intervención}

Se realizaron cuatro ANOVAs con medidas repetidas para analizar los cambios en las actitudes, la conducta percibida y el conocimiento como consecuencia de la intervención didáctica propuesta. En todos estos análisis, se especificó un factor intrasujetos, con dos niveles (pre/post test); y un factor intersujetos definido por la pertenencia al grupo de control/ experimental (Figura 1). Para contrastar la hipótesis de que la matriz de varianzas-covarianzas era circular o esférica se recurrió a la prueba de esfericidad de Mauchly, utilizándose el índice corrector épsilon para aquellos casos en los que no fue posible mantener este supuesto. Como se observa en la Figura 1, la intervención didáctica resultó ser efectiva para todas las 
http://doi.org/10.15359/ree.25-3.11

http://www.una.ac.cr/educare

educare@una.ac.cr

variables objeto de estudio, ya que se obtuvieron incrementos estadísticamente significativos en el grupo experimental. En concreto, la magnitud del efecto resultó ser más alta para el ecoconocimiento, con un $\eta^{2}$ parcial próximo a .28; seguido de la eco-afinidad y la conducta percibida de separación de residuos, con $\eta^{2}$ parciales próximos a .20; y en último lugar el conocimiento sobre la separación de residuos, con un $\eta^{2}$ parcial de .08 .

Figura 1: Evaluación de los cambios en actitudes, conductas y nivel de conocimiento como consecuencia de la intervención didáctica

$$
\begin{gathered}
\text { Eco-afinidad } \\
\mathrm{F}=18.249, \mathrm{p}<.001, \mathrm{n}^{2} \text { parcial }=.200
\end{gathered}
$$

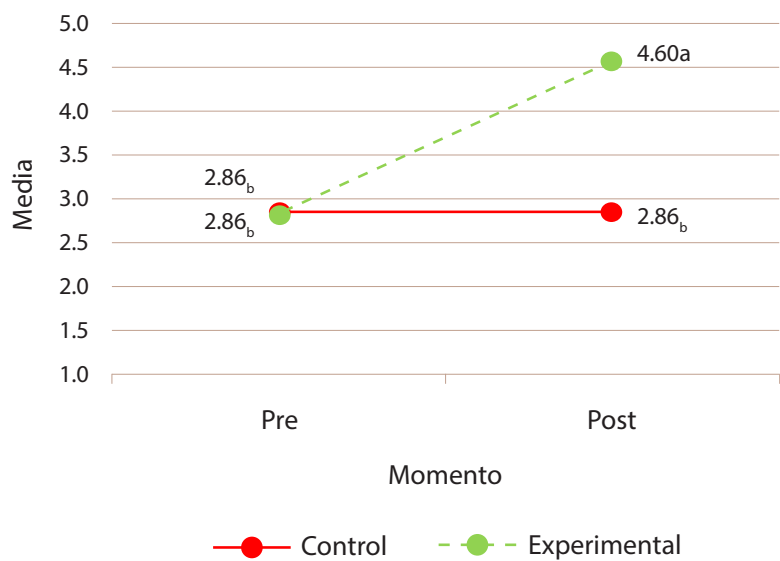

Conducta percibida de separación de residuos $\mathrm{F}=16.533, \mathrm{p}<.001, \eta^{2}$ parcial $=.185$

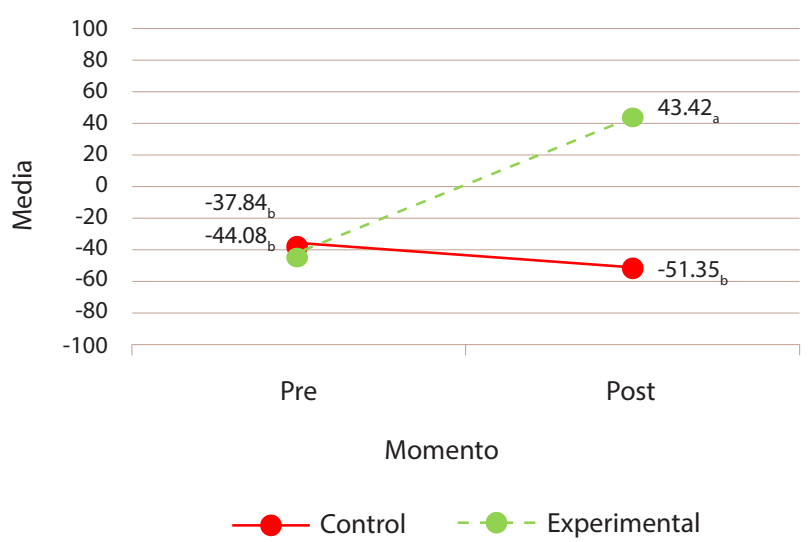

Eco-conocimiento $\mathrm{F}=28.245, \mathrm{p}<.001, \eta^{2}$ parcial $=.279$

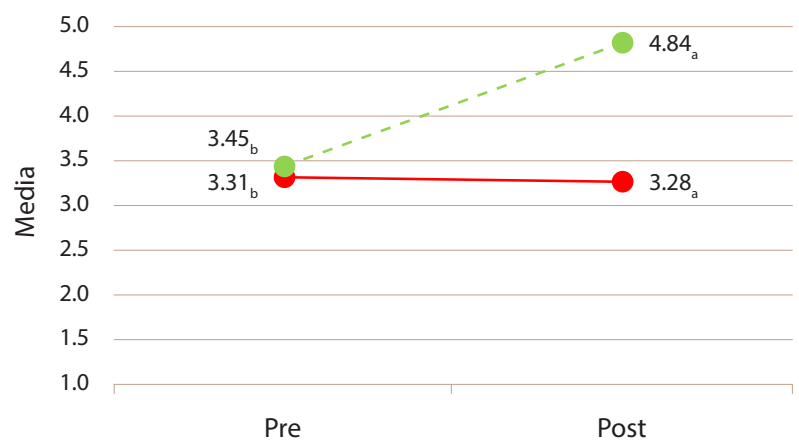

Momento

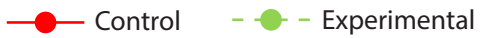

Conocimiento sobre separación de residuos $\mathrm{F}=6.379, \mathrm{p}=.014, \mathrm{\eta}^{2}$ parcial $=.080$

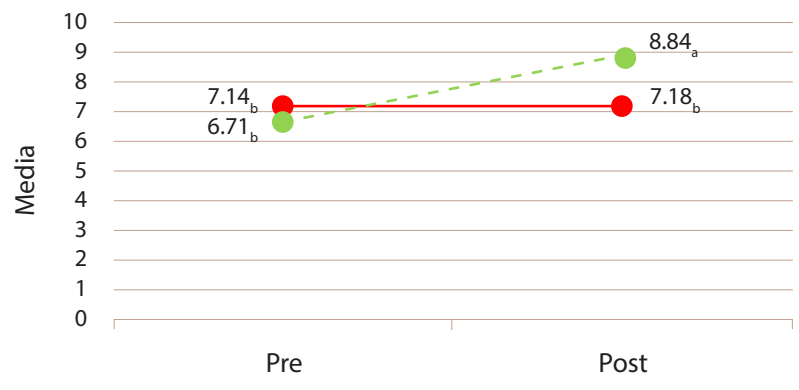

Momento

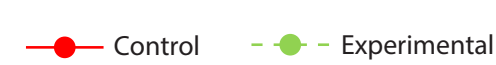

Nota: Las medias que no comparten los subíndices difieren significativamente entre sí $(p<.05)$.

Elaboración propia. 


\section{Discusión}

El presente trabajo ha estudiado las actitudes del alumnado participante hacia el medio ambiente, su percepción sobre la conducta de separación de residuos en el hogar y su nivel de conocimiento respecto a la separación doméstica de residuos. Según indican los resultados de la Tabla 1, las escolares y los escolares analizados mostraron inicialmente una actitud proambiental moderada, una percepción de la conducta de separación de residuos en el ámbito doméstico baja y un nivel de conocimiento notable sobre la adecuada separación de residuos en diferentes contenedores; en la misma línea que otros estudios previos (Perales García y García Perales, 2017; Pérez-Franco et al., 2018).

El objetivo principal fue analizar el efecto de una intervención en el aula diseñada ad hoc sobre las actitudes de escolares hacia el medio ambiente, la percepción de la conducta de reciclaje doméstico y el conocimiento relativo a la separación de residuos en el hogar. En cuanto a las actitudes proambientales del alumnado, se observó cómo las personas participantes pertenecientes al grupo control mostraron valores similares en el pre-test y en el post-test; manifestaron, en ambas ocasiones, una actitud proambiental moderada. Sin embargo, el grupo experimental registró valores significativamente mayores después de la intervención en el aula, mostró una actitud proambiental más elevada; lo que coincide con otros trabajos realizados fuera del entorno español (Torres Rivera et al., 2016). En particular, se observó que el incremento en las actitudes resultó ser mayor en la dimensión de eco-conocimiento que en la de eco-afinidad. Este resultado concuerda con el contenido de la intervención didáctica propuesta, que fue diseñada fundamentalmente para generar un mayor conocimiento de lo que podríamos denominar como conducta ecológica responsable, centrada en el reciclaje. De este modo, podemos inferir que la intervención en el aula ha influido positivamente sobre las actitudes de respeto y cuidado hacia el medio ambiente del alumnado, lo cual confirma la primera hipótesis planteada.

Respecto a la percepción de conducta de separación de residuos en el ámbito doméstico, se pudo comprobar que el grupo control y el experimental no puntuaron estadísticamente diferente en el pre-test. En el caso del grupo experimental, se pudo observar una notable mejoría en su puntuación media en el post-test, lo que ratificaría la segunda hipótesis planteada, en consonancia con los resultados obtenidos por intervenciones similares en el ámbito de la educación ambiental (Bermúdez, 2015). Es importante tener en cuenta que de los resultados de este estudio no se puede afirmar que la intervención influya positivamente sobre las conductas de separación de residuos de las personas participantes, pues no se ha medido la conducta de forma directa, sino la percepción de esta en el ámbito doméstico. El impacto positivo de la intervención realizada en el aula contribuyó a mejorar la percepción de quienes participaron sobre la separación de residuos en sus hogares, lo que podría derivar en un compromiso mayor por su parte en la conducta mencionada e incluso en la influencia de las personas participantes 
http://doi.org/10.15359/ree.25-3.11

http://www.una.ac.cr/educare

educare@una.ac.cr

sobre sus familiares a la hora de llevar a cabo la adecuada separación de residuos antes de desecharlos.

En cuanto al nivel de conocimiento del alumnado respecto a la separación de residuos en diferentes contenedores, se pudo comprobar cómo los escolares y las escolares que formaron el grupo control registraron puntuaciones similares en las dos aplicaciones del cuestionario. Sin embargo, en el grupo experimental se observaron valores significativamente mayores tras la intervención. De este modo, no se pudo rechazar la tercera hipótesis planteada en el presente trabajo, la cual afirmaba que el nivel de conocimiento del alumnado respecto a la adecuada separación de residuos mejoraría tras la intervención en el aula; lo cual concuerda con conclusiones similares a las obtenidas en otros trabajos previos en este ámbito (Alarcón Rojas et al., 2017). No obstante, cabría señalar que, de los tres constructos analizados, el relativo al nivel de conocimiento es el que registró un incremento más moderado. Este resultado podría deberse a que el conocimiento inicial de quienes participaron ya era alto (al ser la separación de residuos una conducta normalizada en España).

\section{Conclusiones}

Las conclusiones de este trabajo deben ser valoradas con prudencia al tratarse de un estudio de caso con una muestra incidental y de conveniencia. En futuros estudios sería conveniente aplicar la metodología experimental presentada en otros contextos diferentes (socioeconómicos, culturales, educativos, etc.). Ello permitiría contrastar si los resultados obtenidos son o no generalizables y, así, validar la intervención didáctica diseñada y crear un protocolo de educación ambiental en escolares de primaria. Asimismo, sería conveniente ampliar las variables implicadas incluyendo la dimensión afectiva, que no ha sido contemplada en este trabajo. Por ejemplo, el grado de conectividad con la naturaleza y su efecto moderador entre las actitudes y las conductas proambientales. Esta variable que refleja las relaciones emocionales entre el yo y la naturaleza (Olivos-Jara et al., 2020) se considera esencial durante la infancia, ya que afecta las relaciones de la niñez hacia el medio ambiente y hacia la naturaleza y su comportamiento futuro como persona adulta (Mustapa et al., 2020). Finalmente, señalar la importancia de realizar una segunda toma de datos en el futuro para verificar si los resultados obtenidos se mantienen a lo largo del tiempo. La escasez de estudios encontrados sobre intervenciones en educación ambiental en el panorama español y, sobre todo su evaluación posterior, debería llevar a reflexionar sobre la necesidad de ahondar en investigaciones que diseñen y evalúen intervenciones en este sentido. Por tanto, resulta imprescindible tratar, desde la escuela, la importancia del cuidado y la conservación del medio ambiente, con intervenciones didácticas que favorezcan la conciencia ecológica del estudiantado, modifiquen la relación entre la sociedad y la naturaleza e impulsen el desarrollo de actitudes, valores y capacidades a favor del medio y que permitan, a sus estudiantes, actuar en consecuencia (Aguilar-Correa et al., 2019). 


\section{Declaración de Material complementario}

Este artículo tiene disponible, como material complementario:

-La versión preprint del artículo en https://doi.org/10.5281/zenodo.4042010

\section{Referencias}

Aguilar-Correa, C., Valencia-Fuentes, C., Huentemilla-Rebolledo, M., Valderrama-González, D., Rojas-Correa, Á., Méndez-Contreras, M. y Tapia-Hernández, C. (2019). Percepción sobre servicios ecosistémicos culturales asociados al bosque nativo por parte de un grupo universitario de estudiantes de pedagogía. Revista Electrónica Educare, 23(3), 1-24. http:// doi.org/10.15359/ree.23-3.19

Alarcón Rojas, J. M., Opayome Montaño, M. C. y Velásquez Camacho, L. Y. (2017). El reciclaje, una estrategia de educación ambiental socialmente responsable. Perspectivas, (7), 60-69. http://revistas.uniminuto.edu/index.php/Pers/article/view/1595)

Bermúdez, A. (2015). Proyectos escolares de reciclaje como estrategia para promover actitudes y conductas proambientales en la educación media. Revista Internacional de Pedagogía y Currículo, 2(1), 49-62. https://cgscholar.com/bookstore/works/los-proyectos-escolaresde-reciclaje-como-estrategia-para-promover-actitudes-y-conductas-proambientales-enla-educacion-media?category id=cgrn-es

Cardoso, C. de A., Silvério, G. B., Guijarro, M. E. C., Antoniassi, B. y Siqueira, M. V. B. M. (2018). Avaliação da concepção ambiental ema do $3^{\circ}$ ano do ensino médio: Um estudo de caso em Bauru/SP. Fronteiras: Journal of Social, Technological and nvironmental Science, 6(3), 305-319.

Centro de Investigaciones Sociológicas. (2010). Medio ambiente (II) (International Social Survey Programme). Estudio N.o 2.837. http://www.cis.es/cis/opencm/ES/1 encuestas/estudios/ ver.jsp?estudio $=11404$

Cerrillo, P. C. (2010). Sobre lectura, literatura y educación. Miguel Ángel Porrúa.

Demoly, K. R. do A. y Santos, J. S. B. Dos (2018). Learning, environmental education and school: Ways of en acting in the experience of students and teachers. Ambiente \& Sociedade, (21), 1-20. http://doi.org/10.1590/1809-4422asoc0087r2vu1811ao

Dunlap, R. E. y Jones, R. E. (2002). Environmental concern: Conceptual and measurement issues. En R. E. Dunlap y W. Michelson (Eds.), Handbook of environmental sociology (pp. 482-524). Greenwood Press. 
http://doi.org/10.15359/ree.25-3.11

http://www.una.ac.cr/educare

educare@una.ac.cr

Etxaniz, X. (2011). La transmisión de valores en la literatura, desde la tradición oral hasta la LIJ actual. Ocnos: Revista de Estudios sobre Lectura, (7), 73-83. http://doi.org/10.18239/ ocnos 2011.07.06

Flórez-Yepes, G. Y., Rincón-Santamaría, A., Cardona, P. S. y Gallego, F. A. (2018). Herramientas de aprendizaje para favorecer la educación ambiental. Caso de estudio Fundación Niños de Los Andes sede Manizales, Colombia. Revista Electrónica Educare, 22(2), 1-21. http://doi. org/10.15359/ree.22-2.5

Galvis Riaño, C. J., Perales Palacios, F. J. y Ladino Ospina, Y. (2019). ¿Cómo implementan la educación ambiental en sus aulas profesores de centros educativos rurales colombianos? Contextos Educativos, (23), 101-123. http://doi.org/10.18172/con.3519

Hernández, B. y Suárez, E. (2006). El alcance del ambientalismo: Análisis de algunas consistencias e inconsistencias del comportamiento proambiental. En M. Amérigo y B. Cortés (Eds.), Entre la persona y el entorno. Intersticios para la investigación medioambiental (pp. 59-80). Editorial Resma.

Jiménez-Denis, O., Villalón-Legrá, G. y Evora-Larios, O. E. (2017). La educación para la percepción de riesgos de desastres como prioridad del trabajo educativo en la escuela cubana. Revista Electrónica Educare, 21(3), 1-12. http://doi.org/10.15359/ree.21-3.20

Lima, M.-L. y Branco, C. (2018). Recycling for my neighbourhood? Using place identity and social norms to promote pro-environmental behaviour. Psyecology, 9(1), 1-32. http://doi.org/10. $\underline{1080 / 21711976.2017 .1412574}$

López-Miguens, M. J., González-Vázquez, E., García-Rodríguez, M. J. y Álvarez-González, P. (2014). Empirical evidence of the influence of environmental concern on the ecological behaviour of the consumer. Psyecology, 5(1), 58-90. http://doi.org/10.1080/21711976.2014.881666

Mahasneh, R. A., Romanowski, M. H. y Dajani, R. B. (2017). Reading social stories in the community: A promising intervention for promoting children's environmental knowledge and behavior in Jordan. The Journal of Environmental Education, 48(5), 334-346. http://doi. org/10.1080/00958964.2017.1319789

More, C. M. (2012). Social stories ${ }^{\mathrm{TM}}$ and young children: Strategies for teachers. Intervention in School and Clinic, 47(3), 167-174. http://doi.org/10.1177/1053451211423816

Moreno, I., Amérigo, M. y García, J. A. (2016). Design and application of an environmental attitudes scale in primary education. Psyecology, 7(1), 64-88. http://doi.org/10.1080/2171 $\underline{1976.2015 .1114217}$ 
http://doi.org/10.15359/ree.25-3.11

Moreno-Fernández, O. (2017). ¿Qué sabes de la contaminación? Estudio de las ideas previas en alumnado de educación primaria. Revista Electrónica de Enseñanza de las Ciencias, 16(3), 502-515. http://reec.uvigo.es/volumenes/volumen16/REEC $16 \quad 3 \quad 5$ ex963.pdf

Mustapa, N. D., Maliki, N. Z., Aziz, N. F. y Hamzah, A. (2020). Assessing children's connectedness to nature: A mixed method study. Environment-Behaviour Proceedings Journal, 5(14), 3-9. https://doi.org/10.21834/ebpj.v5i14.2282

Olivos-Jara, P., Segura-Fernández, R., Rubio-Pérez, C. y Felipe-García, B. (2020). Biophilia and biophobia as emotional attribution to nature in children of 5 years old. Frontiers in Psychology, 11(511), 1-14. https://doi.org/10.3389/fpsyg.2020.00511

Ordóñez-Díaz, M. M., Montes-Arias, L. M. y Garzón-Cortés, G. del P. (2018). Importancia de la educación ambiental en la gestión del riesgo socio-natural en cinco países de América Latina y el Caribe. Revista Electrónica Educare, 22(1), 1-19. http://doi.org/10.15359/ree.22-1.17

Passafaro, P. y Livi, S. (2017). Comparing determinants of perceived and actual recycling skills: The role of motivational, behavioral and dispositional factors. The Journal of Environmental Education, 48(5), 347-356. http://doi.org/10.1080/00958964.2017.1320961

Paula-Acosta, C. A., Pérez-López, J. y Sierra-Socorro, J. J. (2019). La educación ambiental con enfoque integrador. Una experiencia en la formación inicial de profesores de matemática y física. Revista Electrónica Educare, 23(1), 1-22. http://doi.org/10.15359/ree.23-1.10

Perales García, R. y García Perales, R. (2017). Educación en valores y atención a la diversidad: Una propuesta práctica desde la educación ambiental. En A. Rodríguez-Martín (Comp.), Prácticas innovadoras inclusivas. Retos y oportunidades (pp. 2413-2421). Universidad de Oviedo. http:// digibuo.uniovi.es/dspace/bitstream/10651/50349/4/EducacionenValores.pdf

Pérez-Franco, D., de Pro-Bueno A. J. y Pérez-Manzano, A. (2018). Actitudes ambientales al final de la ESO. Un estudio diagnóstico con alumnos de secundaria de la región de Murcia. Revista Eureka sobre Enseñanza y Divulgación de las Ciencias, 15(3), 1-17. https://doi.org/10.25267/ Rev Eureka ensen divulg cienc.2018.v15.i3.3501

Programa de las Naciones Unidas para el Desarrollo. (2015). Objetivos de desarrollo sostenible. https://www.undp.org/content/undp/es/home/sustainable-development-goals.html

Severiche-Sierra, C., Gómez-Bustamante, E. y Jaimes-Morales, J. (2016). La educación ambiental como base cultural y estrategia para el desarrollo sostenible. Telos: Revista de Estudios Interdisciplinarios en Ciencias Sociales, 18(2), 266-281. https://dialnet.unirioja.es/servlet/ articulo?codigo $=5655393$ 
http://doi.org/10.15359/ree.25-3.11

http://www.una.ac.cr/educare

educare@una.ac.cr

Steg, L. y Vlek, C. (2009). Encouraging pro-environmental behaviour: An integrative review and research agenda. Journal of Environmental Psychology, 29(3), 309-317. https://doi. org/10.1016/j.jenvp.2008.10.004

Stern, P. C. (2000). Toward a coherent theory of environmentally significant behavior. Journal of Social Issues, 56(3), 407-424. https://doi.org/10.1111/0022-4537.00175

Thorn, C. y Bogner, F. X. (2018). How environmental values predict acquisition of different cognitive knowledge types with regard to forest conservation. Sustainability, 10(7), 1-11. http://doi.org/10.3390/su10072188

Torres Rivera, L. B., Mesina Calderón, N., Salamanca Salazar, B. y Sepúlveda Sepúlveda, C. (2016). Efectos de la enseñanza interdisciplinaria en la educación ambiental sobre los conocimientos, valores y actitudes ambientales de estudiantes de segundo ciclo básico (Los Ángeles, región del Biobío, Chile). Revista Complutense de Educación, 27(3), 1139-1155. http://doi.org/10.5209/rev RCED.2016.v27.n3.47551

Vanegas-Rico, M.-C., Corral-Verdugo, V., Ortega-Andeane, P. y Bustos-Aguayo, J.-M. (2018). Intrinsic and extrinsic benefits as promoters of pro-environmental behaviour. Psyecology, 9(1), 33-54. http://doi.org/10.1080/21711976.2017.1390034

Viladrich, C., Angulo-Brunet, A. y Doval, E. (2017). A journey around alpha and omega to estimate internal consistency reliability. Anales de Psicología, 33(3), 755-782. http://doi.org/10.6018/ analesps.33.3.268401

White, R. L., Eberstein, K. y Scott, D. M. (2017). Birds in the playground: Evaluating the effectiveness of an urban environmental education project in enhancing school children's awareness, knowledge and attitudes towards local wildlife. PLOS ONE, 13(3), 1-23. http:// doi.org/10.1371/journal.pone.0193993 